\title{
Cascade of failures in coupled network systems with multiple support-dependent relations
}

\author{
Jia Shao ${ }^{1}$, Sergey V. Buldyrev ${ }^{2,1}$, Shlomo Havlin ${ }^{3}$ and H. Eugene Stanley ${ }^{11}$ \\ ${ }^{1}{ }^{1}$ Center for Polymer Studies and Department of Physics, \\ Boston University, Boston, Massachusetts 02215, USA \\ ${ }^{2}$ Department of Physics, Yeshiva University, \\ 500 West 185th Street, New York, New York 10033, USA. \\ ${ }^{3}$ Minerva Center and Department of Physics, \\ Bar-Ilan University, 52900 Ramat-Gan, Israel
}

(Dated: Printed: October 23, 2018)

\begin{abstract}
We study, both analytically and numerically, the cascade of failures in two coupled network systems A and B, where multiple support-dependent relations are randomly built between nodes of networks A and B. In our model we assume that each node in one network can function only if it has at least a single support node in the other network. If both networks $\mathrm{A}$ and $\mathrm{B}$ are Erdős-Rényi networks, A and B, with (i) sizes $N^{A}$ and $N^{B}$, (ii) average degrees $a$ and $b$, and (iii) $c_{0}^{A B} N^{B}$ support links from network A to B and $c_{0}^{B A} N^{B}$ support links from network B to A, we find that under random attack with removal of fractions $\left(1-R^{A}\right) N^{A}$ and $\left(1-R^{B}\right) N^{B}$ nodes respectively, the percolating giant components of both networks at the end of the cascading failures, $\mu_{\infty}^{A}$ and $\mu_{\infty}^{B}$, are given by the percolation laws $\mu_{\infty}^{A}=R^{A}\left[1-\exp \left(-c_{0}^{B A} \mu_{\infty}^{B}\right)\right]\left[1-\exp \left(-a \mu_{\infty}^{A}\right)\right]$ and $\mu_{\infty}^{B}=R^{B}\left[1-\exp \left(-c_{0}^{A B} \mu_{\infty}^{A}\right)\right]\left[1-\exp \left(-b \mu_{\infty}^{B}\right)\right]$. In the limit of $c_{0}^{B A} \rightarrow \infty$ and $c_{0}^{A B} \rightarrow \infty$, both networks become independent, and the giant components are equivalent to a random attack on a single Erdős-Rényi network. We also test our theory on two coupled scale-free networks, and find good agreement with the simulations.
\end{abstract}




\section{INTRODUCTION}

In recent years, there has been extensive effort to study and understand the properties of complex networks. Research has mainly focused on properties of single networks which do not interact or depend on other networks [1 14]]. Recently, the robustness of two interdependent coupled networks has been studied [15, 16]. In interdependent networks, the failures of nodes in one network, A, will cause failures of dependent nodes in the other network, B, and vice versa. This process occurs recursively, and leads to a cascade of failures. It has been shown both analytically and numerically that the robustness of two interdependent networks is significantly lower compared to that of a single network [15]. Furthermore, the percolation transition in coupled networks is first order compared to the known second order transition in a single network [15, 16].

Previous studies on two interdependent coupled networks are restricted by the condition, that to function each node in network $\mathrm{A}$ depends on one and only one node in network $\mathrm{B}$ and vice versa [15]. However, in the real world, this assumption may not be valid. A single node in network A may depend on more than one node in network B and will function as long as one of the support nodes in network B is still connected. Similarly, a node in network B may depend on more than one support nodes in network A. As long as one of the support nodes functions, the node in network B will also function.

Examples of such systems include the coupled power grid network and the communication network which controls the power grid, where both networks depend on each other. In general, one power station provides power to more than one communication stations, and one communication station controls more than one power stations. As long as a communication station can obtain power from one power station, it can function properly. On the other hand, one communication station is sufficient in sending control signals which make one power station function properly. However without any power, the communication station will fail, and without control the power station will stop working. Indeed in the 2003, due to failure of some power stations in Italy, the communication control system was damaged. This damage caused further fragmentation of the power grid, which finally led to a blackout in a sinificant part of Italy.

Under random attack, which is characterized by random removal of nodes in one or both networks, the coupled network systems demonstrate significantly different behavior from 
that of a single network [15]. The failures of nodes in network A can lead to the failures of dependent nodes in network $\mathrm{B}$, and the failures of nodes in network $\mathrm{B}$ can produce a feedback on network A leading to further failures in network A. This process can occur recursively and can lead to a cascade of failures.

We provide a theoretical framework for understanding the robustness of interdependent networks with a random number of support and dependent relationships. Our theory agrees well with the numerical simulations of several model network systems, including coupled Erdős-Rényi (ER) [17] and coupled scale-free (SF) [2] networks. Our work extends previous works on coupled networks [15, 16] from one-to-one dependent-support relation to multiple Poissonian dependent-support relation. Our model can help to further understand real-life coupled network systems, where complex dependence-support relations may exist.

We define the stable state to be the state when the cascade of failures ends. We show that for two coupled ER networks the giant components of both networks in the stable state follow a simple law, which is equivalent to random percolation of a single network in the limit of a large number of support links. Our theory is relevant to a broad class of real-world interdependent network systems.

The paper is organized as follows. In Sec. II, we explain the model of the cascade of failures with random support-dependent relations. In Sec. III, we derive analytically the process of the failure cascade. In Sec. IV, we present numerical tests on coupled ER and SF networks.

\section{THE MODEL}

We assume two networks $\mathrm{A}$ and B of sizes $N^{A}$ and $N^{B}$ and with given degree distributions, $P^{A}(k)$ and $P^{B}(k)$, of "intra-links" connecting nodes in the same network (Fig. 1). The dependency relation is represented by a link connecting the support node in one network and the dependent node in the other network ("inter-links"). The inter-links between network $\mathrm{A}$ and network $\mathrm{B}$ are random and uni-directional. Initially (stage $n=0$ of the failure cascade), there are $c_{0}^{B A} N^{A}$ inter-links distributed randomly from nodes in network $\mathrm{B}$ to nodes in network A, representing the dependencies of node in network A on nodes in network B. Similarly, there are $c_{0}^{A B} N^{B}$ inter-links from nodes in network A to nodes in network B, representing dependencies of nodes in network B on nodes in network A. The dependent- 
support relations are random i.e., for each inter-link, the support node and dependent node

in the two networks are chosen randomly. $c_{0}^{B A}$ and $c_{0}^{A B}$ are the initial mean degrees of the corresponding inter-links for networks $\mathrm{A}$ and $\mathrm{B}$ respectively.

In our model, one node in either network A or network B can have zero, one or several support nodes in the other network. We assume that to function each node in network A requires at least one support node in network B, and vice versa. Nodes in each network which are not connected to the giant components of that network by the intra-links, and nodes without support inter-links, are considered to be not functioning and regarded as failed nodes.

The attack on the network is represented by a random removal of fraction $1-R^{A}$ nodes in network $\mathrm{A}$ and $1-R^{B}$ in network $\mathrm{B}$, where in general, $R^{A} \neq R^{B}$. The process of the cascade of failures is demonstrated in Fig. 1. With $N^{A}=N^{B}=7$, we show the case of random removal of one node in network $\mathrm{A}\left(R^{A}=6 / 7\right)$ and one node in network $\mathrm{B}\left(R^{B}=6 / 7\right)$. At each stage of the cascade of failures, both networks will experience further failures. Without loss of generality, we assume the random attack on network A occurs before that on network B. Thus, when we analyze the first stage of the cascade of failures in network A, all the support inter-links from network B are considered functioning. At each stage, the nodes which do not have any support inter-links from the other network, and the nodes which are separated from the giant component of the network, are considered to have failed. This process will continue until no further node failure in either network occurs.

\section{ANALYTICAL SOLUTION}

The stable state of the two stable connected giant components in both networks are usually reached after several stages in the cascade of failures. We define $c_{n}^{A B}$ and $c_{n}^{B A}$ as the average number of support inter-links remaining in the stage $n$ of the cascade of failures of network $\mathrm{A}$ and network $\mathrm{B}$ respectively.

In each stage of the cascade of failures, we analyze first network A then network B. Such a procedure does not have any affect on the final result of the failure cascade. While considering network A at stage $n$, we assume all the inter-links $\left(c_{n-1}^{B A}\right)$ from network $\mathrm{B}$ to network $\mathrm{A}$ of the previous stage are working. When considering network $\mathrm{B}$ at stage $n$, we use the updated $c_{n}^{A B}$ after the $n$th stage of network A. 


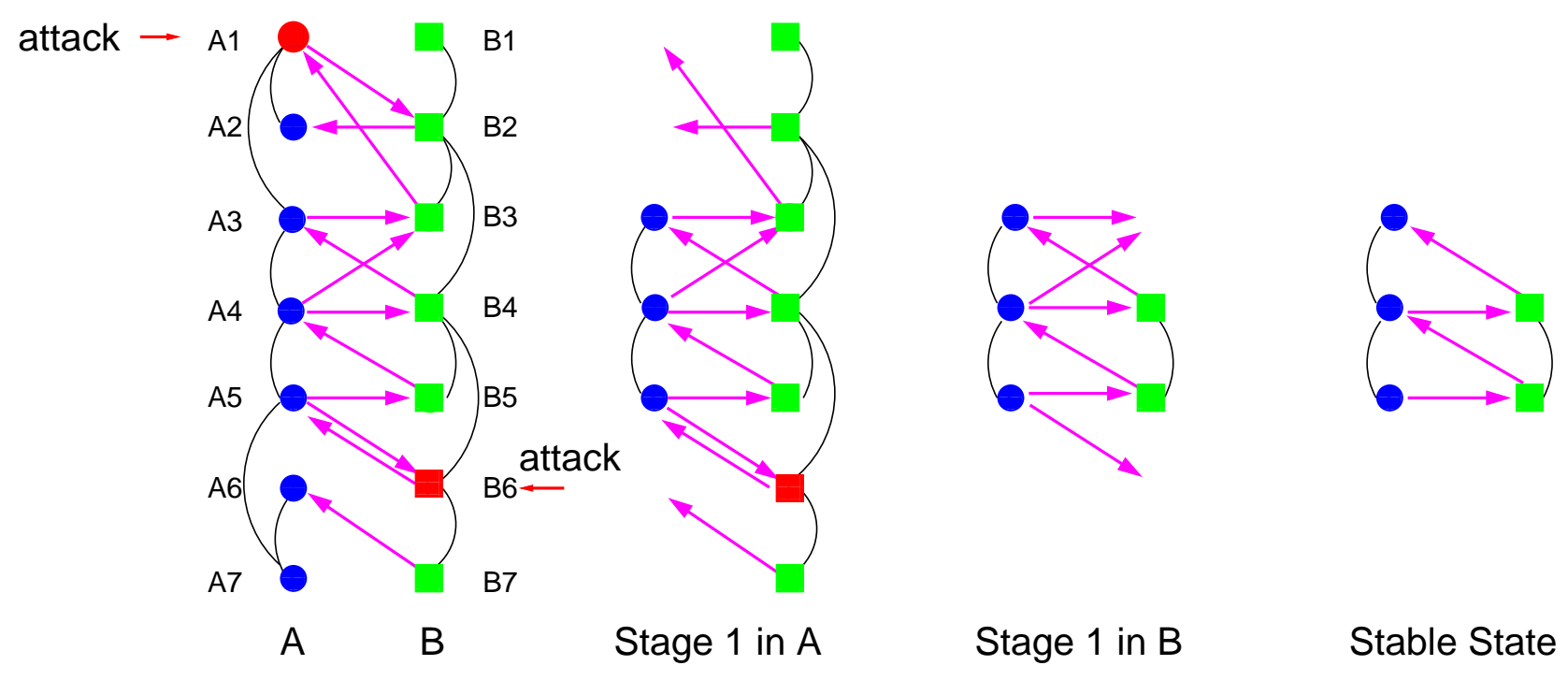

FIG. 1: (Color online) Demonstration of the stages of the cascade of failures in coupled network A and B of size $N^{A}=N^{B}=7$. Curves represent intra-links within the network, while arrows (directed links) represent the inter-links connecting a support node in one network to the dependent node in the other network. Among the total 12 directed links, half of them $c_{0}^{B A} N^{A}=6$ represent the support from nodes in network B to nodes in network A (arrow from nodes in network B to nodes in network A). The rest $c_{0}^{A B} N^{B}=6$ links represent the support from nodes in network A to nodes in network B. The support-dependent relations between nodes in network A and network B are random. Initially, the attack is on node $A_{1}$ (shown in red) in network $\mathrm{A}$ and node $B_{6}$ (shown in red) in network B. The failed nodes are removed from the plot. In the first stage of the cascade of failures in network $\mathrm{A}, A_{1}$ fails because of removal, node $A_{7}$ fails because of it has no support inter-links, $A_{2}$ and $A_{6}$ fail because of separation from the giant component of network $\mathrm{A}$. All the failed nodes will lead to failures of inter-links starting from them. Since we assume that the attack on network $\mathrm{A}$ occurs before that on network $\mathrm{B}$, the inter-link from $B_{6}$ to $A_{5}$ is considered to be functional. In the first stage of the cascade of failures in network B, we first remove inter-links connecting network $\mathrm{B}$ to non-giant-component nodes in network $\mathrm{A}$ ( $B_{3}$ to $A_{1}, B_{2}$ to $A_{2}, B_{7}$ to $\left.A_{7}\right)$. Next, node $B_{6}$ fails because of the attack and nodes $B_{1}, B_{2}$ and $B_{7}$ also fail because of no support. $B_{3}$ fails because it becomes separated from the giant component (nodes $B_{4}$ and $B_{5}$ ) of network B. Finally, after removing inter-links connecting from network A to non-giant-component nodes in network $\mathrm{B}\left(A_{3}\right.$ to $B_{3}, A_{4}$ to $B_{3}$ and $A_{5}$ to $\left.B_{6}\right)$, the coupled network system reaches a stable state after one step in the cascade of failures, since all nodes in both giant components are connected and each node have at least one support node from the other network. 
Since, in our model we randomly set up $c_{0}^{A B} N^{B}$ inter-links from network A to network B and $c_{0}^{B A} N^{A}$ inter-links from network B to network $\mathrm{A}$, the degree distributions of inter-links of both networks follow Poisson statistics. There exist nodes in network A and network B without support initially (nodes $A_{7}$ and $B_{1}$ in Fig.1). These nodes are regarded as failed and need to be considered in addition to the random removal of nodes.

At stage 1 , after removal of a fraction $1-R^{A}$ of nodes in network $\mathrm{A}$, taking into account also the nodes with zero inter-links at stage 0 , the result is equivalent to a random removal of a fraction $1-p_{1}^{A}\left(R^{A}, c_{0}^{B A}\right)$ of nodes in network $\mathrm{A}$. The giant component of network $\mathrm{A}$ will constitute a fraction $g_{1}^{A}\left(p_{1}^{A}, c_{0}^{B A}\right)$ of the remaining $p_{1}^{A} N^{A}$ nodes of network A. Thus, the fraction $\mu_{1}^{A}$ of the giant component with respect to the $N^{A}$, the original size of network A, is

$$
\mu_{1}^{A}=p_{1}^{A}\left(R^{A}, c_{0}^{B A}\right) g_{1}^{A}\left(p_{1}^{A}, c_{0}^{B A}\right)
$$

Since we assume that all support links from network B exist, we use $c_{0}^{B A}$.

The failures of $\left(1-\mu_{1}^{A}\right) N^{A}$ nodes in network A will lead to failures of support inter-links from network A to network B. The working support inter-links from network A to network $\mathrm{B}, c^{A B}$, will decrease from $c_{0}^{A B}$ to $c_{1}^{A B}\left(\mu_{1}^{A}\right)$.

Next, at the first stage of cascade of failures in network $\mathrm{B}$, a fraction $1-R^{B}$ of nodes in network B will fail because of the initial attack. Combining this fraction with the fraction of nodes in network B having zero degree of support links after the first stage of cascade of failures in network $\mathrm{A}$, the joint effect is equivalent to a random removal of a fraction $1-p_{1}^{B}\left(R^{B}, c_{1}^{A B}\right)$ of nodes in network $\mathrm{B}$. The giant component of network $\mathrm{B}$ will constitute a fraction $g_{1}^{B}\left(p_{1}^{B}, c_{1}^{A B}\right)$ of $p_{1}^{B} N^{B}$ nodes. Thus the fraction of the giant component of network $\mathrm{B}$ after the 1st stage of cascade of failures is

$$
\mu_{1}^{B}=p_{1}^{B}\left(R^{B}, c_{1}^{A B}\right) g_{1}^{B}\left(p_{1}^{B}, c_{1}^{A B}\right) .
$$

The number of support links $c^{B A}$ then will be reduced from $c_{0}^{B A}$ to $c_{1}^{B A}\left(\mu_{1}^{B}\right)$.

The 2nd stage of the cascade of failures in network $A$ is equivalent to the first stage in network $\mathrm{A}$ with the updated $c_{1}^{B A}$ replacing $c_{0}^{B A}$. Accordingly, the 2 nd stage of cascade failures in network $\mathrm{B}$ is equivalent to the first stage in network $\mathrm{B}$ with the updated $c_{2}^{A B}$. During the cascade of failures, $c_{n}^{A B}$ and $c_{n}^{B A}$ decrease as $n$ increases.

In general, the $n$th stage of the cascade in network $\mathrm{A}$ is equivalent to the 1 st stage of the cascade in network $\mathrm{A}$ with $c_{0}^{B A}$ replaced by $c_{n-1}^{B A}$. Similarly, the $n$th stage of the cascade in 
network $\mathrm{B}$ is equivalent to the 1 st stage of the cascade in network $\mathrm{B}$ with $c_{1}^{A B}$ replaced $c_{n}^{A B}$. The general forms of the giant components of both networks at stage $n$ of the cascade of failures can be expressed as

$$
\left\{\begin{array}{l}
\mu_{n}^{A}=p_{n}^{A}\left(R^{A}, c_{n-1}^{B A}\right) g_{n}^{A}\left(p_{n}^{A}, c_{n-1}^{B A}\right), \\
\mu_{n}^{B}=p_{n}^{B}\left(R^{B}, c_{n}^{A B}\right) g_{n}^{B}\left(p_{n}^{B}, c_{n}^{A B}\right),
\end{array}\right.
$$

where $p_{n}^{A}$ and $p_{n}^{B}$ are the equivalent fractions of nodes in network $\mathrm{A}$ and network $\mathrm{B}$ respectively after random removal, and $g_{n}^{A}$ and $g_{n}^{B}$ are the fractions of the giant components in the remaining $p_{n}^{A}$ and $p_{n}^{B}$ fraction of nodes. The key to the analytical solution of this process is to find the way how $c_{n}^{A B}$ and $c_{n}^{B A}$ decrease with the cascade stage $n$.

Next, we will use the apparatus of generating functions [20] to derive the analytical forms of $\mu_{n}^{A}$ and $\mu_{n}^{B}, c_{n}^{A B}$ and $c_{n}^{B A}$. The generating functions of the degree distribution $P^{A}(k)$ of network $\mathrm{A}$ and $P^{B}(k)$ of network $\mathrm{B}$ are

$$
\left\{\begin{array}{l}
G_{A 0}(x) \equiv \Sigma_{k=0}^{\infty} P^{A}(k) x^{k} \\
G_{B 0}(x) \equiv \Sigma_{k=0}^{\infty} P^{B}(k) x^{k}
\end{array}\right.
$$

Analogously, the generating functions of the underlying branching processes are

$$
\left\{\begin{array}{l}
G_{A 1}(x) \equiv G_{A 0}^{\prime}(x) / G_{A 0}^{\prime}(1), \\
G_{B 1}(x) \equiv G_{B 0}^{\prime}(x) / G_{B 0}^{\prime}(1) .
\end{array}\right.
$$

After random removal of a fraction $1-p$ of nodes, the remaining $p$ fraction of the network will have different degree distribution. The new generation functions $G_{0}$ and $G_{1}$ will be [18, 19]

$$
\left\{\begin{aligned}
G_{A 0}(x, p) & =G_{A 0}(1-p(1-x)), \\
G_{B 0}(x, p) & =G_{B 0}(1-p(1-x)), \\
G_{A 1}(x, p) & \equiv G_{A 1}(1-p(1-x)), \\
G_{B 1}(x, p) & \equiv G_{B 1}(1-p(1-x)) .
\end{aligned}\right.
$$

Randomly connecting $c^{B A} N^{A}$ support links from network B to network A, the degree distribution of the inter-links in network A follows a Poisson distribution with average degree $c^{B A}$

$$
\tilde{P}^{A}(k)=\frac{\left[c^{B A}\right]^{k}}{k !} e^{-c^{B A}}
$$

Similar, for network B,

$$
\tilde{P}^{B}(k)=\frac{\left[c^{A B}\right]^{k}}{k !} e^{-c^{A B}}
$$


During the process of the cascade of failures, because the support-dependent relations are uncorrelated with the network properties of network A and network B, $\tilde{P}^{A}(k)$ and $\tilde{P}^{B}(k)$ will remain Poisson distributions with the new $c_{n}^{B A}$ and $c_{n}^{A B}$, which decrease as $n$ increases.

Initially, there will be a fraction $\tilde{P}^{A}(0)=e^{-c^{B A}}$ of nodes in network A which do not have any support links from network B. Since the attack on the $1-R^{A}$ fraction of nodes from network A is random, there will be overlap between the attack and the initially not working nodes (without support links) in network A. The joint effect is equivalent to a random removal of a fraction $1-R^{A}\left(1-e^{-c^{B A}}\right)$ of nodes in network $\mathrm{A}$. What happens in network $\mathrm{B}$ is similar to network A with $R^{A}$ replaced by $R^{B}$ and $c^{B A}$ replaced by $c^{A B}$. Thus

$$
\left\{\begin{array}{l}
p_{n}^{A}=R^{A}\left(1-e^{-c_{n-1}^{B A}}\right), \\
p_{n}^{B}=R^{B}\left(1-e^{-c_{n}^{A B}}\right),
\end{array}\right.
$$

where $c_{n-1}^{B A}$ and $c_{n}^{A B}$ are average degree of inter-links of network A and network B at the end of stage $n-1$ and $n$ respectively.

According to the results on single networks [18, 19], after random removal of a fraction $1-p^{A}$ (or $1-p^{B}$ ) of nodes, the fractions of nodes that belong to the giant components of the remaining network $\mathrm{A}$ or network $\mathrm{B}$, which have $p_{n}^{A}$ and $p_{n}^{B}$ fractions of nodes respectively, are

$$
\left\{\begin{array}{l}
g^{A}\left(p_{n}^{A}\right)=1-G_{A 0}\left(f_{n}^{A}, p_{n}^{A}\right), \\
g^{B}\left(p_{n}^{B}\right)=1-G_{B 0}\left(f_{n}^{B}, p_{n}^{B}\right) .
\end{array}\right.
$$

where $f_{n}^{A}$ and $f_{n}^{B}$ satisfy transcendental equations

$$
\left\{\begin{array}{l}
f_{n}^{A}=G_{A 1}\left(f_{n}^{A}, p_{n}^{A}\right), \\
f_{n}^{B}=G_{B 1}\left(f_{n}^{B}, p_{n}^{B}\right) .
\end{array}\right.
$$

Thus $\mu_{n}^{A}$ and $\mu_{n}^{B}$, the fractions relative to their original sizes of giant components of network A and network B [18, 19] are

$$
\left\{\begin{array}{l}
\mu_{n}^{A}=p_{n}^{A} g^{A}\left(p_{n}^{A}\right), \\
\mu_{n}^{B}=p_{n}^{B} g^{B}\left(p_{n}^{B}\right) .
\end{array}\right.
$$

Accordingly, $c^{A B}$ and $c^{B A}$ follow the relations

$$
\left\{\begin{array}{l}
c_{n}^{A B}=c_{0}^{A B} \mu_{n}^{A}, \\
c_{n}^{B A}=c_{0}^{B A} \mu_{n}^{B} .
\end{array}\right.
$$


When the cascade of failures process stops, $f_{n}^{A}, f_{n}^{B}, p_{n}^{A}, p_{n}^{B}, c_{n}^{A B}, c_{n}^{B A}, \mu_{n}^{A}$ and $\mu_{n}^{B}$ all reach the constant values, $f_{\infty}^{A}, f_{\infty}^{B}, p_{\infty}^{A}, p_{\infty}^{B}, c_{\infty}^{A B}, c_{\infty}^{B A}, \mu_{\infty}^{A}$ and $\mu_{\infty}^{B}$ respectively. In principle, these final values can be found from the set of equations:

$$
\left\{\begin{array}{l}
f_{\infty}^{A}=G_{A 1}\left(f_{\infty}^{A}, p_{\infty}^{A}\right), \\
f_{\infty}^{B}=G_{B 1}\left(f_{\infty}^{B}, p_{\infty}^{B}\right), \\
p_{\infty}^{A}=R^{A}\left(1-e^{-c_{\infty}^{B A}}\right), \\
p_{\infty}^{B}=R^{B}\left(1-e^{-c_{\infty}^{A B}}\right), \\
c_{\infty}^{A B}=c_{0}^{A B} g^{A}\left(p_{\infty}^{A}\right)=c_{0}^{A B}\left[1-G_{A 0}\left(f_{\infty}^{A}, p_{\infty}^{A}\right)\right], \\
c_{\infty}^{B A}=c_{0}^{B A} g^{B}\left(p_{\infty}^{B}\right)=c_{0}^{B A}\left[1-G_{B 0}\left(f_{\infty}^{B}, p_{\infty}^{B}\right)\right], \\
\mu_{\infty}^{A}=p_{\infty}^{A} g^{A}\left(p_{\infty}^{A}\right), \\
\mu_{\infty}^{B}=p_{\infty}^{B} g^{B}\left(p_{\infty}^{B}\right) .
\end{array}\right.
$$

The functional forms of $G_{A 1}, G_{B 1}, G_{A 0}$ and $G_{B 0}$ can be complicated, thus Eqs.(14) can only be solved numerically for most cases, including coupled SF networks. However, for ER networks, $G_{0}(x)$ and $G_{1}(x)$ have the same simple form [20],

$$
G_{0}(x)=G_{1}(x)=e^{\langle k\rangle(x-1)},
$$

where for network $\mathrm{A},\langle k\rangle=a$ and for network $\mathrm{B},\langle k\rangle=b$. Thus, the above process of the cascade of failures can be significantly simplified. Eqs.(10) can be reduced to

$$
\left\{\begin{array}{l}
g^{A}\left(p^{A}\right)=1-f^{A} \\
g^{B}\left(p^{B}\right)=1-f^{B}
\end{array}\right.
$$

Excluding $p_{\infty}^{A}, p_{\infty}^{B}, c_{\infty}^{A B}, c_{\infty}^{B A}, \mu_{\infty}^{A}$ and $\mu_{\infty}^{B}$ from Eqs.(14), for the stable state of two coupled ER networks, we get a system of two equations with two remaining unknowns, $f_{\infty}^{A}$ and $f_{\infty}^{B}$

$$
\left\{\begin{array}{l}
f_{\infty}^{A}=G_{A 1}\left(f_{\infty}^{A}, R^{A}\left(1-e^{-c_{\infty}^{B A}}\right)\right)=e^{a R^{A}\left(f_{\infty}^{A}-1\right)\left(1-e^{-c_{\infty}^{B A}}\right)}=e^{a R^{A}\left(f_{\infty}^{A}-1\right)\left(1-e^{c_{0}^{B A}\left(f_{\infty}^{B}-1\right)}\right)}, \\
f_{\infty}^{B}=G_{B 1}\left(f_{\infty}^{B}, R^{B}\left(1-e^{-c_{\infty}^{A B}}\right)\right)=e^{b R^{B}\left(f_{\infty}^{B}-1\right)\left(1-e^{-c_{\infty}^{A B}}\right)}=e^{b R^{B}\left(f_{\infty}^{B}-1\right)\left(1-e^{c_{0}^{A B}\left(f_{\infty}^{A}-1\right)}\right)} .
\end{array}\right.
$$

The solutions of Eqs.(17) for $f_{\infty}^{A}$ and $f_{\infty}^{B}$ can be used to calculate the stable mutually connected giant components of both networks as

$$
\left\{\begin{array}{l}
\mu_{\infty}^{A}=p_{\infty}^{A} g^{A}\left(p_{\infty}^{A}\right)=R^{A}\left(1-e^{-c_{\infty}^{B A}}\right)\left(1-f_{\infty}^{A}\right)=-\ln \left(f_{\infty}^{A}\right) / a, \\
\mu_{\infty}^{B}=p_{\infty}^{B} g^{B}\left(p_{\infty}^{B}\right)=R^{B}\left(1-e^{-c_{\infty}^{A B}}\right)\left(1-f_{\infty}^{B}\right)=-\ln \left(f_{\infty}^{B}\right) / b .
\end{array}\right.
$$

From Eqs.(18), using Eqs.(13), we can derive the percolation law for the fractions of the stable giant components of both coupled ER networks 


$$
\begin{gathered}
\mu_{\infty}^{A}=R^{A}\left(1-e^{-c_{0}^{B A} \mu_{\infty}^{B}}\right)\left(1-e^{-a \mu_{\infty}^{A}}\right), \\
\mu_{\infty}^{B}=R^{B}\left(1-e^{-c_{0}^{A B} \mu_{\infty}^{A}}\right)\left(1-e^{-b \mu_{\infty}^{B}}\right) .
\end{gathered}
$$

Eq.(19) and Eq.(20) are simple and can be related to the theory of random percolation of a single ER network [21, 22], for which the fraction of the giant component is $\mu_{\infty}=$ $R\left(1-e^{-\langle k\rangle \mu_{\infty}}\right)$. The coupled ER networks bring new terms $1-e^{-c_{0}^{B A} \mu_{\infty}^{B}}$ and $1-e^{-c_{0}^{A B} \mu_{\infty}^{A}}$. In the limit of $c_{0}^{A B} \rightarrow \infty$ (or $\left.c_{0}^{B A} \rightarrow \infty\right)$, the giant component of network $\mathrm{B}$ (or network $\mathrm{A}$ ) does not depend on the other network and behaves similarly to the random percolation of a single network.

From Eqs.(19) and (20), we find $\mu_{\infty}^{A}$ and $\mu_{\infty}^{B}$ for a given set of parameters $R^{A}, R^{B}, c_{0}^{B A}$ and $c_{0}^{A B}$. However, for some values of $R^{A}, R^{B}, c_{0}^{B A}$ and $c_{0}^{A B}$, the solutions for $\mu_{\infty}^{A}$ and $\mu_{\infty}^{B}$ between 0 and 1 may not exist. There exist critical thresholds of $R^{A}, R^{B}, c_{0}^{B A}$ and $c_{0}^{A B}$ above which the two coupled ER networks have non-zero mutually connected giant components (see Fig,2). They are represented as $R_{c}^{A}, R_{c}^{B}, c_{c}^{B A}$ and $c_{c}^{A B}$. These values can be solved by finding the tangent point of the two curves $\left(\mu_{\infty}^{A}\right.$ is plotted as a function of $\mu_{\infty}^{B}$ as shown in Fig(2) represented by Eqs.(19) and (20). The thresholds can be found from the tangential condition

$$
\left.\left.\frac{d \mu_{\infty}^{A}}{d \mu_{\infty}^{B}}\right|_{E q .(19)} \frac{d \mu_{\infty}^{B}}{d \mu_{\infty}^{A}}\right|_{E q .(20)}=1,
$$

together with Eqs.(19) and (20).

\section{NUMERICAL SIMULATIONS}

Next, we compare our theoretical results obtained in Sec. III to results of numerical simulations. We begin with comparing the simulations of the stages of the failure cascade in coupled ER networks with our theoretical predictions. In all our simulations, we use $N^{A}=N^{B}=10^{6}$. Fig. 3] shows $\mu_{n}^{A}$ and $\mu_{n}^{B}$ as a function of $n$ for $a=b=4, c_{0}^{A B}=c_{0}^{B A}=4$,

$R^{B}=1$ and for different values of $R^{A}$. One sees very good agreement between the theory and the simulations. Close to $R_{c}^{A}$, both $\mu_{n}^{A}$ and $\mu_{n}^{B}$ show large fluctuations between different realizations (shown in Figs. 3c and 3d). The random realizations split into two classes: one that converges to a non-zero giant component for both networks and the other that 


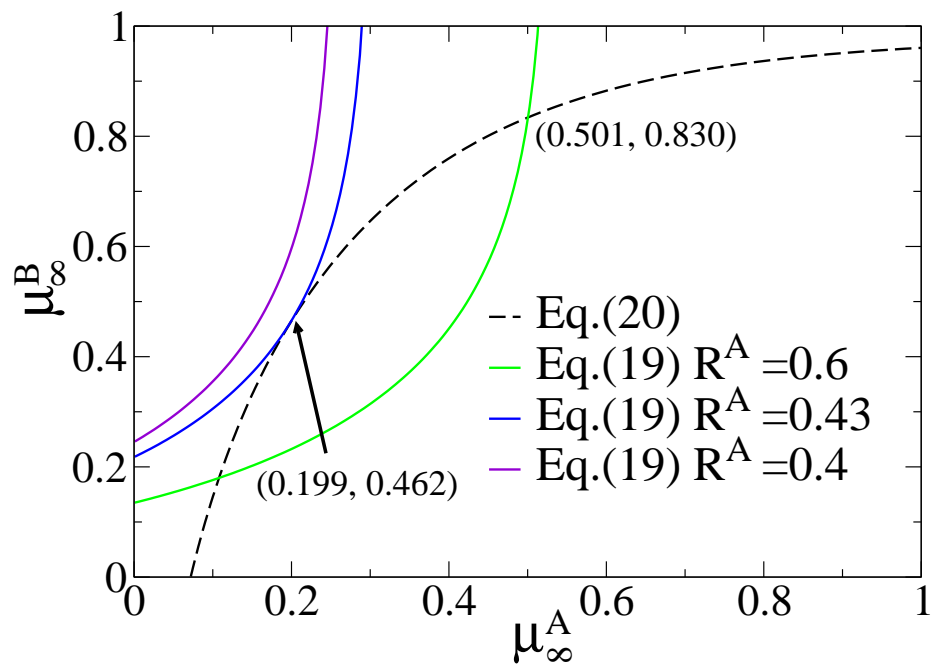

FIG. 2: Demonstration of the functional relation between $\mu_{\infty}^{B}$ and $\mu_{\infty}^{A}$ in Eq.(19) and Eq.(20) for a system of two coupled ER networks with $a=b=4, c_{0}^{A B}=c_{0}^{B A}=4$, and $R^{B}=1$ with different values of $R^{A}$. Since we use $R^{B}=1$, at different $R^{A}$, the relation between $\mu_{\infty}^{B}$ and $\mu_{\infty}^{A}$ given by Eq.(20) remains the same (shown by the dashed line). Eq.(19) with $R^{A}=0.6,0.43$ and 0.4 are shown. One can see that when $R^{A}<0.43$, there exists no solution $\left(\mu_{\infty}^{A}, \mu_{\infty}^{B}\right)$ for Eq.(19) and Eq.(20). The value $1-R_{c}^{A} \equiv 1-0.43=0.57$ represents the maximum fraction of nodes in network A one can randomly remove at the initial stage of the cascade of failures for which the non-zero giant components of both networks still exist at the stable state. The abrupt fragmentation of the stable giant components at $R^{A}<R_{c}^{A}$ represents the first order nature of the percolation phase transition.

results in a complete fragmentation. The agreement between the simulations and theoretical predictions is also good for different values of $R^{B}, a, b$ and $c_{0}^{A B}$ and $c_{0}^{B A}$.

In Fig. 4, we compare the theoretical predictions and simulations of the giant components at stages of the cascade of failures for a system of two coupled SF networks with $\lambda^{A}=\lambda^{B}=$ 2.5, $c_{0}^{A B}=c_{0}^{B A}=4, R^{B}=1$ and different values of $R^{A}$. Similarly, we obtain agreement between the theoretical predictions and the simulations. Close to $R_{c}^{A}$, both $\mu_{n}^{A}$ and $\mu_{n}^{B}$ of different realizations show large fluctuations and the random realizations also split into two classes. We also simulated other values of $R^{B}, \lambda^{A}, \lambda^{B}, c_{0}^{A B}$ and $c_{0}^{B A}$ and found very good agreement between and theoretical predictions and simulations.

The fractions of the giant components of both network $\mathrm{A}\left(\mu_{\infty}^{A}\right)$ and network $\mathrm{B}\left(\mu_{\infty}^{A}\right)$ in the 

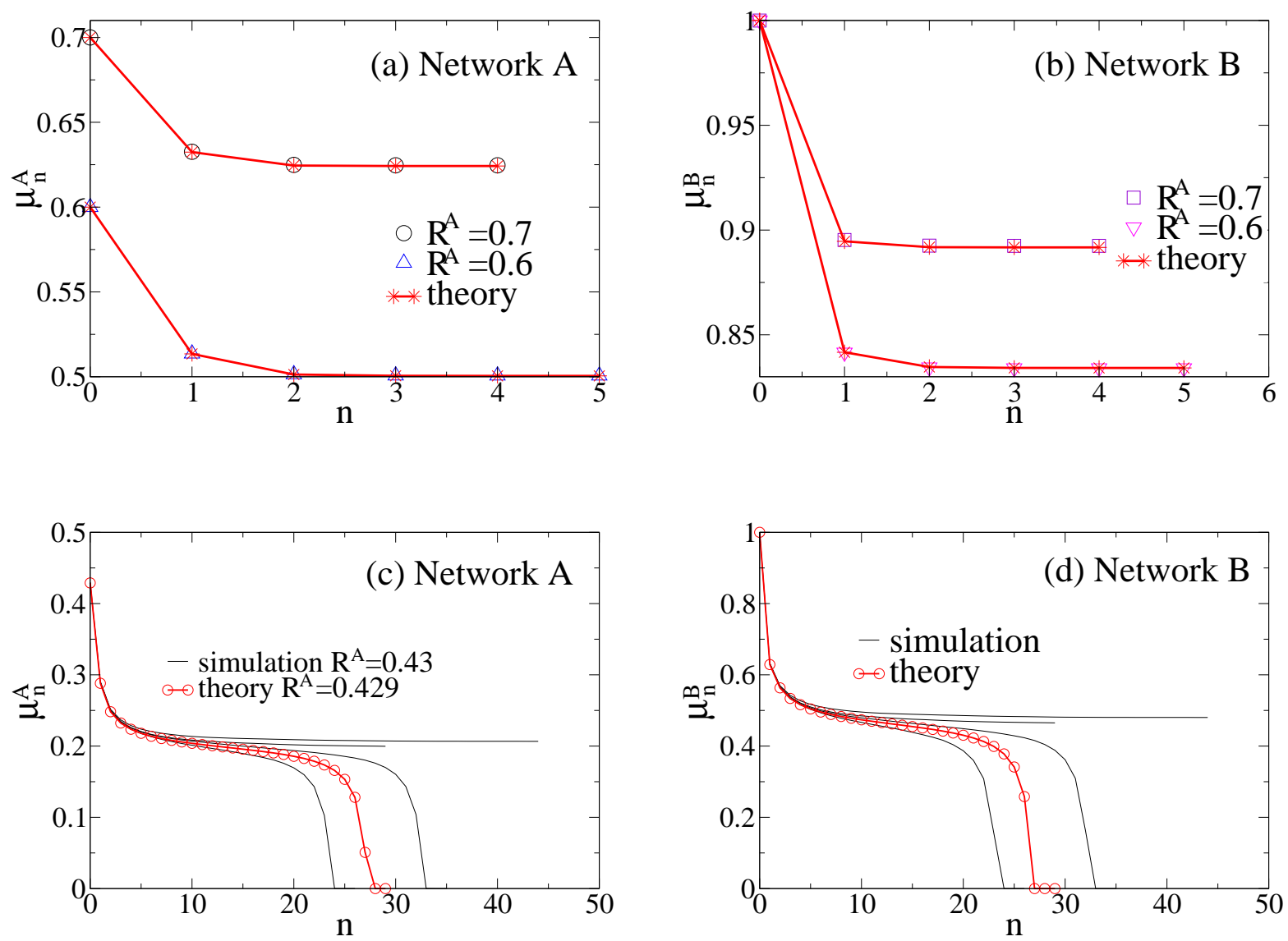

FIG. 3: The case of coupled ER networks. Comparison between the theoretical predictions, obtained from Eqs.(16), and Eqs.(12-13), and numerical simulations with $N^{A}=N^{B}=10^{6}, a=b=4$, $c_{0}^{A B}=c_{0}^{B A}=4, R^{B}=1$ and several values of $R^{A}$. (a) and (b) show $\mu_{n}^{A}$ and $\mu_{n}^{B}$ at different stages $n$ of the cascade of failures for $R^{A}=0.7$ and 0.6 above $R_{c}^{A} \approx 0.43$ for both theory (lines) and simulations (symbols). One can see that both $\mu_{n}^{A}$ and $\mu_{n}^{B}$ approach a stable value $\mu_{\infty}^{A}$ and $\mu_{\infty}^{B}$ at the end of the cascade of failures. The agreement between theory and numerical simulations is very good. (c) and (d) show $\mu_{n}^{A}$ and $\mu_{n}^{B}$ at different stages $n$ of the cascade of failures for $R^{A} \approx R_{c}^{A}$. The bare lines represent several realizations of the simulations and the lines with symbols represent the theoretical predictions. One can see that for the early stages (small $n$ ) the agreement is good, however at large $n$ the deviation due to random fluctuations in the actual fraction of the giant component starts to increase. The random realizations split into two classes: one that converges to a non-zero giant component for both networks and the other that results in a complete fragmentation. 

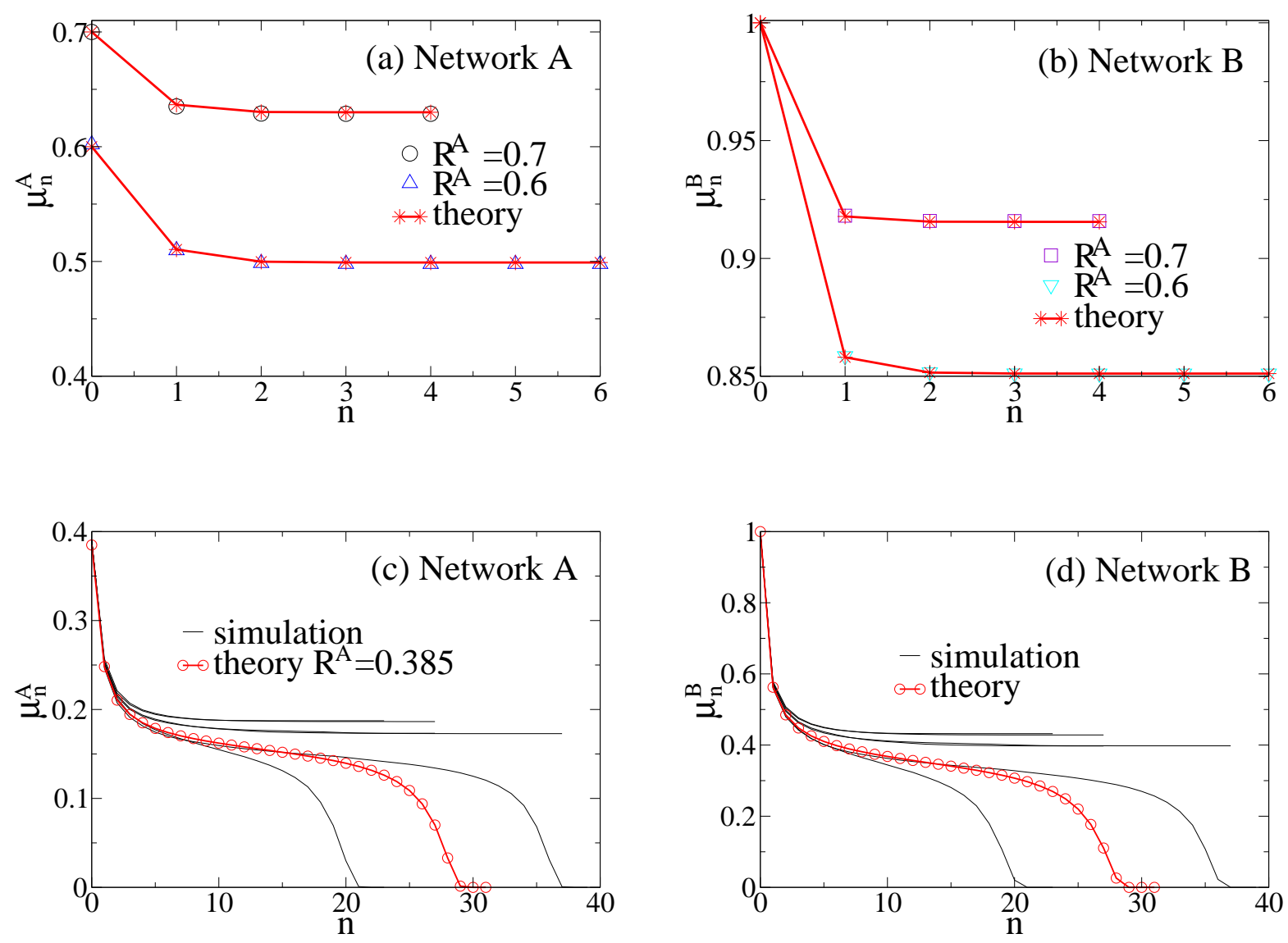

FIG. 4: The case of coupled SF networks. Comparison between the theoretical predictions, obtained from Eqs.(10), and Eqs.(11,13), and numerical simulations with $N^{A}=N^{B}=10^{6}, \lambda^{A}=\lambda^{B}=2.5$, $c_{0}^{A B}=c_{0}^{B A}=4, R^{B}=1$ and different values of $R^{A}$. (a) and (b) show $\mu_{n}^{A}$ and $\mu_{n}^{B}$ at different stages $n$ of the cascade of failures for $R^{A}=0.7$ and 0.6 above $R_{c}^{A} \approx 0.385$ for both theory (lines) and simulations (symbols). Similar to Fig. 3 , one can see that both $\mu_{n}^{A}$ and $\mu_{n}^{B}$ approach a stable value $\mu_{\infty}^{A}$ and $\mu_{\infty}^{B}$ at the end of cascade failures. The agreement between the theory and numerical simulations is very good. (c) and (d) show $\mu_{n}^{A}$ and $\mu_{n}^{B}$ at different stages $n$ of the cascade of failures for $R^{A} \approx R_{c}^{A}$. Bare lines represent several realizations of the simulations and the lines with symbols represent the theoretical predictions. One can see that for the early stages the agreement is good, however at large $n$ the deviation due to random fluctuations in the actual fraction of the giant component increase. The random realizations split into two classes: one that converges to a nonzero giant component for both networks and the other that results in a complete fragmentation. For coupled SF networks, at $R^{A}=R_{c}^{A}$, the fluctuations of both $\mu_{n}^{A}$ and $\mu_{n}^{B}$ seem to be relatively larger than that of coupled ER networks, due to the existence of large degree nodes in SF networks. 

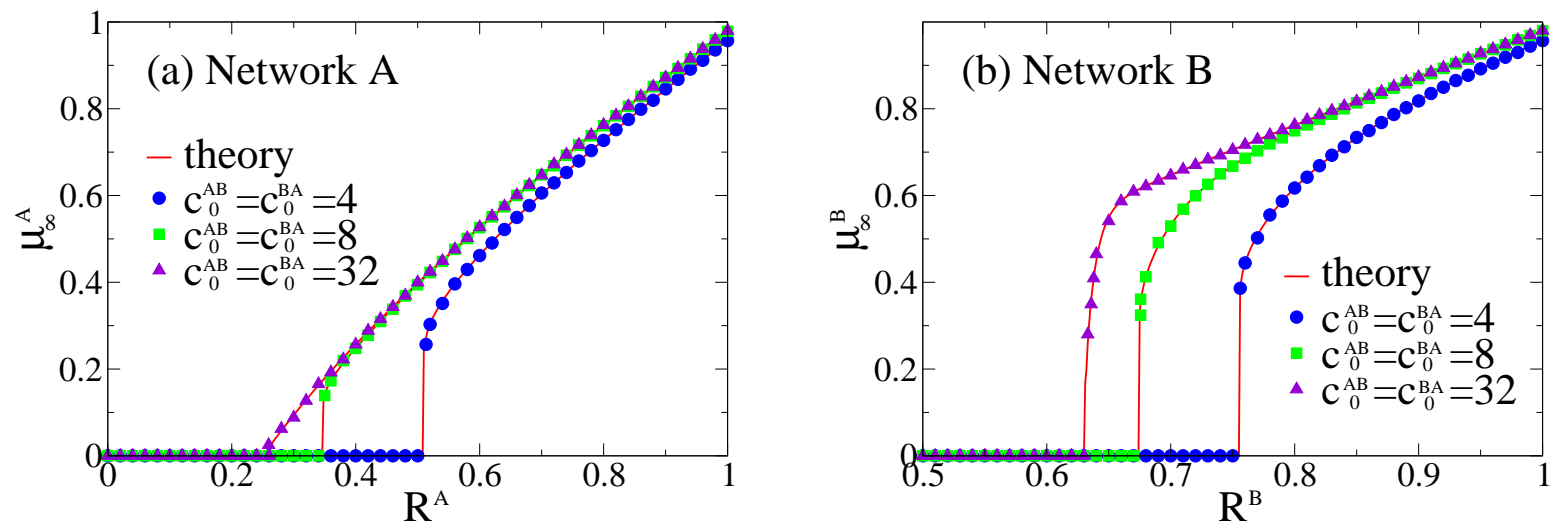

FIG. 5: (a) $\mu_{\infty}^{A}$ and (b) $\mu_{\infty}^{B}$ in the stable states as a function of $R^{A}$ and $R^{B}$ for coupled ER networks $\mathrm{A}$ and B with $N^{A}=N^{B}=10^{6}, a=b=4, c_{0}^{A B}=c_{0}^{B A}=4$ and $1-R^{A}=2\left(1-R^{B}\right)$. Several curves for $c_{0}^{A B}=c_{0}^{B A}=4,8$ and 32 are shown. The theory (lines) fits very well with the simulation results (symbols). One can see that for a given set of $c_{0}^{A B}$ and $c_{0}^{B A}$ there exist critical thresholds $R_{c}^{A}$ and $R_{c}^{B}$, below which both networks will collapse and have no stable non-zero giant components. The value of $R_{c}^{A}$ approaches the critical threshold of random percolation $(r=1 / a=0.25)$ of a single network for large values of $c_{0}^{A B}$ and $c_{0}^{B A}$. The initial attack on network $\mathrm{B}$ is smaller than that on network $\mathrm{A}$ and thus $R_{c}^{B}>R_{c}^{A}$.

stable state can be found from Eqs.(19) and (20) for coupled ER networks. We solve these equations numerically for different $R^{A}$ and $R^{B}$, and compare the theoretical predictions with the simulation results (Fig. 5). For simplicity, we assume $a=b=4$ and that the initial fraction of nodes affected by the random attack in network $\mathrm{A}$ is twice as large as that in network $\mathrm{B}\left(1-R^{A}=2\left(1-R^{B}\right)\right)$. We test different average degrees of inter-links for both networks $c_{0}^{A B}=c_{0}^{B A}$.

In Fig. 5, we present results for the giant components of both networks as a function of $R^{A}$ and $R^{B}$. For different sets of $c_{0}^{A B}$ and $c_{0}^{B A}$, we find again that the theory fits well with simulation results. One can see the critical $R_{c}^{A}$ and $R_{c}^{B}$, which are the minimum fractions of both networks needed to be kept at the beginning of the cascade of failures in order to have non-zero connected giant components of both networks at the stable state. At $R_{c}^{A}$ and $R_{c}^{B}$, both $\mu_{\infty}^{A}$ and $\mu_{\infty}^{B}$ show an abrupt change from a finite fraction $\left(\mu_{c}^{A}\right.$ and $\left.\mu_{c}^{B}\right)$ to zero. As $c_{0}^{A B}$ and $c_{0}^{B A}$ increase, $R_{c}^{A}$ approaches the critical threshold of random percolation of a single ER network, which is $1 / a$. As expected for single networks, $\mu_{c}^{A}$ and $\mu_{c}^{B}$ approach 0 and a 


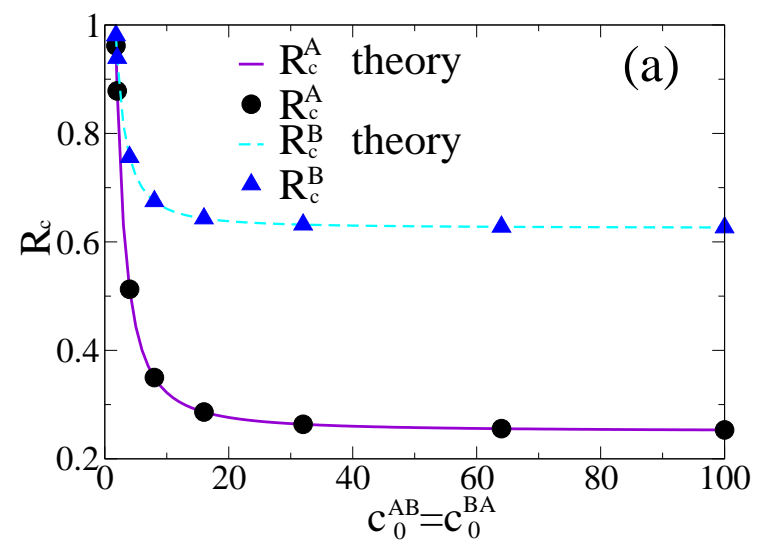

FIG. 6: The dependences of $R_{c}$ (a) and $\mu_{c}$ (B) on $c_{0}^{A B}=c_{0}^{B A}$ for coupled ER networks with $N^{A}=N^{B}=10^{6}, a=b=4$, and $1-R^{A}=2\left(1-R^{B}\right)$. In (a), the critical initial fraction of the network $\mathrm{A}, R_{c}^{A}$, and the critical initial fraction of the network $\mathrm{B}, R_{c}^{B}$, are shown as a function of $c_{0}^{A B}=c_{0}^{B A}$. The theory (full line and dashed lines) fits well the simulation results (symbols). $R_{c}^{A}$ approaches the critical threshold $(1 / a)$ of random percolation for a single network, as predicted by Eqs.(19) and (20). In (b), the giant component of both network $\mu_{c}^{A}$ and $\mu_{c}^{B}$ are shown as a function of $c_{0}^{A B}=c_{0}^{B A}$ at $R_{c}^{A}$ and $R_{c}^{B}$. The theory (full line and dashed lines) fits well the simulation results (symbols). One can see that for large $c_{0}^{A B}=c_{0}^{B A}, \mu_{c}^{A}$ and $\mu_{c}^{B}$ both approach zero as expected for a single network. However, $\mu_{c}^{A}$ and $\mu_{c}^{B}$ will never reach zero for finite $c_{0}^{A B}$ and $c_{0}^{B A}$, the phase transition thus remain a first order.

second order phase transition exists for large $c_{0}^{A B}$ and $c_{0}^{B A}$. However, for finite $c_{0}^{A B}$ and $c_{0}^{B A}$ the changes of $\mu_{\infty}^{A}$ and $\mu_{\infty}^{B}$ are not continuous at $R_{c}^{A}$ and $R_{c}^{B}$, indicating a first order phase transition. This result is predicted by Eqs.(19) and (20). We find that the theory fits well with the simulation results for the entire range of $R_{c}^{A}$ and $R_{c}^{B}$ for different values of $c_{0}^{A B}$ and $c_{0}^{B A}$.

Next, we study the dependence of $R_{c}^{A}$ and $R_{c}^{B}, \mu_{c}^{A}$ and $\mu_{c}^{B}$ on $c_{0}^{A B}$ and $c_{0}^{B A}$ (see Fig [6). For simplicity and for comparing with our earlier cases, we use the same set of parameter

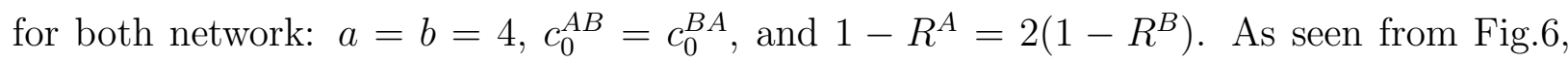
the theory fit well the numerical simulations. For large $c_{0}^{A B}=c_{0}^{B A}$, one can see that $R_{c}^{A}$ approaches the random percolation threshold $1 / a$ on a single ER network. This behavior indicates that when one network A has enough support from network B and vice versa, both networks will behave as if they are independent. Indeed for large $c_{0}^{A B}$ and $c_{0}^{B A}$, at $R_{c}^{A}$ and 
$R_{c}^{B}$, the stable giant components of both networks $\mu_{c}^{A}$ and $\mu_{c}^{A}$ approach zero as expected for a second order percolation phase transition. However, as seen from Eqs.(19) and (20), for finite values of $c_{0}^{A B}$ and $c_{0}^{B A}$, neither $\mu_{c}^{A}$ nor $\mu_{c}^{B}$ is zero. This result supports the existence of a first order phase transition for the entire range of $c_{0}^{A B}$ and $c_{0}^{B A}$. Good agreement between theory and simulations, and similar behavior of $R_{c}^{A}, R_{c}^{B}, \mu_{c}^{A}$ and $\mu_{c}^{A}$ as a functions of $c_{0}^{A B}$ and $c_{0}^{B A}$ have been found for other sets of parameters.

\section{CONCLUSIONS AND DISCUSSIONS}

In this paper, we extend previous works [15, 16] on the cascade of failures on interdependent networks by considering random support-dependent relations between two coupled network systems. Our theory is in excellent agreement with the numerical simulations on coupled Erdős-Rényi (ER) and coupled scale-free (SF) networks systems. For coupled ER networks, the percolation law for the giant components of both networks have a simple form, which in the limit of large number of supports gives the percolation law for single networks. Only in the limit of a large number of support is the percolation transition of second order, while in general case, the coupled network show a first order phase transition. Our model can help to further understand real-life coupled network systems, where complex dependence-support relations exists. Recently, a complementary approach to study the robustness of coupled networks system has been proposed [23], which is based on a quite different assumption about the way networks are coupled. In contrast to our case where $p_{c}$ increases due to coupling, in their case $p_{c}$ decreases. Note that there are also recent efforts to study the robustness of single networks [24-26] undergoing targeted percolation, which correlates with the topology of the network. In the same spirit, our work can be extended to study the robustness of coupled networks under non-random percolation. A first attempt in this direction for interdependent networks can be found in Ref. [27].

\section{Acknowledgments}

We wish to thank the ONR, DTRA, EU project Epiwork, and the Israel Science Foundation for financial support. S.V.B. thanks the Office of the Academic Affairs of Yeshiva University for funding the Yeshiva University high performance computer cluster and ac- 
knowledges the partial support of this research through the Dr. Bernard W. Gamson Computational Science Center at Yeshiva College. 
[1] D. J. Watts and S. H. Strogatz, Nature (London) 393, 440442 (1998).

[2] A. L. Barabási and R. Albert, Science 286, 509(1999).

[3] R. Pastor-Satorras and A. Vespignani, Evolution and Structure of the Internet: A Statistical Physics Approach (Cambridge University Press, 2006).

[4] S. N. Dorogovtsev and J. F. F. Mendes, Evolution of Networks: from Biological nets to the Internet and $W W W$ (Oxford University Press, New York, 2003).

[5] R. Cohen, K. Erez, D. ben-Avraham, and S. Havlin, Phys. Rev. Lett. 85, 4626 (2000).

[6] L. A. Braunstein, Z. Wu, Y. Chen, S. V. Buldyrev, T. Kalisky, S. Sreenivasan, R. Cohen, E. Lopez, S. Havlin, and H. E. Stanley, International Journal of Bifurcation and Chaos 17, 2215 (2007).

[7] J. Shao, S. Havlin, and H. E. Stanley, Phys. Rev. Lett. 103, 018701 (2009).

[8] M. E. J. Newman, S. H. Strogatz, D. J. Watts, Phys. Rev. E 64, 026118 (2001).

[9] M. E. J. Newman, Networks: An Introduction, (Oxford University Press, USA 2010).

[10] R. Cohen and S. Havlin, Complex Networks: Structure, Robustness and Function, (Cambridge University Press, Cambridge, 2010).

[11] S. Boccaletti, V. Latora, Y. Moreno, M. Chavez, D.-U. Hwang, Physics Reports 424, 175(2006).

[12] G. Caldarelli and A. Vespignani, Large scale structure and dynamics of complex networks, (World Scientific, Singapore, 2007).

[13] A. Barrat, M. Barthélemy, and A. Vespignani, Dynamical processes on complex networks (Cambridge University Press, England, 2008).

[14] D. S. Callaway, M. E. J. Newman, S. H. Strogatz and D. J. Watts, Phys. Rev. Lett. 85, $5468(2000)$.

[15] S. V. Buldyrev, R. Parshani, G. Paul, H. E. Stanley, and S. Havlin, Nature (London) 464, $1025(2010)$.

[16] R. Parshani, S. V. Buldyrev, and S. Havlin, Phys. Rev. Lett. 105, 048701(2010).

[17] P. Erdős and A. Rényi, Publ. Math. 6, 290 (1959); Publ. Math. Inst. Hung. Acad. Sci. 5, 17 (1960).

[18] J. Shao, S. V. Buldyrev, R. Cohen, M. Kitsak, S. Havlin, and H. E. Stanley, Europhys. Lett. 
84, 48004 (2008).

[19] J. Shao, S. V. Buldyrev, L. A. Braunstein, S. Havlin, and H. E. Stanley, Phys. Rev. E 80, 036105 (2009).

[20] M. E. J. Newman, Phys. Rev. E 66, 016128 (2002).

[21] B. Bollobás, Random Graphs (Academic, London, 1985).

[22] Fractals and Disordered Systems, Edited by A. Bunde and S. Havlin (Springer, 1996).

[23] E. A. Leicht and R. M. D'Souza, arXiv: cond-mat/0907.0894.

[24] L. K. Gallos, R. Cohen, P. Argyrakis, A. Bunde, and S. Havlin, Phys. Rev. Lett 94, 188701 (2005).

[25] A. A. Moreira, J. S. Andrade, Jr., H. J. Herrmann, and J. O. Indekeu, Phys. Rev. Lett. 102, 018701 (2009).

[26] H. Hooyberghs, B. V. Schaeybroeck, A. A. Moreira, J. S. Andrade, Jr., H. J. Herrmann, and J. O. Indekeu, Phys. Rev. E 81, 011102 (2010).

[27] X. Huang, J. Gao, S. V. Buldyrev, S. Havlin, and H. E. Stanley, arXiv: 1010.2160. 